\title{
Numerical relativity: maximizing the scientific payoff from gravitational wave detection
}

\section{Saul A. Teukolsky}

Saul A. Teukolsky, "Numerical relativity: maximizing the scientific payoff from gravitational wave detection," Proc. SPIE 4856, Gravitational-Wave Detection, (26 February 2003); doi: 10.1117/12.459025

SPIE Event: Astronomical Telescopes and Instrumentation, 2002, Waikoloa, Hawai'i, United States 


\title{
Numerical Relativity: Maximizing the Scientific Payoff From Gravitational Wave Detection
}

\author{
Saul A. Teukolsky ${ }^{a}$ \\ ${ }^{a}$ Departments of Physics and Astronomy, Cornell University, Ithaca, NY 14853
}

\begin{abstract}
For many of the proposed LIGO sources, we are currently unable to produce reliable theoretical waveforms or event rates. Usually the difficulty is in performing numerical simulations. These problems seriously undermine our ability to extract science from LIGO, or in some cases even to detect sources at all. We describe two sources where these problems are present: the merger of a binary black hole system, and a newborn neutron star unstable to r-modes. We explain the origin of the difficulties, and how they might be overcome.
\end{abstract}

Keywords: Einstein's equations, gravitational waves, black holes, neutron stars

\section{INTRODUCTION}

Gravitational wave detectors like LIGO are poised to begin serious scientific data taking. Over the past few years, many possible sources have been discussed. Strategies have been refined to search for these sources in the data. However, for a large fraction of the scientifically most important sources, our theoretical understanding is so limited that we risk being unable to confront theory with the observations, or perhaps even to detect these sources in the first place. In many of these cases the science is well understood, but numerical simulations are needed to determine waveforms or predict event rates. It is difficulties in carrying out these simulations that are holding us up. Until these difficulties are overcome, we will be unable to derive the full scientific payoff from instruments like LIGO.

In this paper I will discuss two examples of source simulations where we are struggling. I will explain why the problems are difficult, and why we are optimistic that solutions can be found. Success, however, will require a significant investment of resources in source simulations that require large scale computing. Given the size of the LIGO and LISA experiments, such an investment in theoretical support is well justified to maximize the scientific payoff.

\section{BLACK HOLE MERGERS}

A prime target for LIGO is the coalescence of binary black hole systems. There are three characteristic regimes during a merger: (i) the initial inspiral; (ii) the nonlinear merger; and (iii) the final ringdown.

During the initial inspiral the binary system progresses through a sequence of quasiequilibrium circular orbits, with the radius slowly decreasing as the system loses energy via gravitational waves. The binary is well described by the post-Newtonian approximation, giving quite accurate predictions of waveforms. These predictions can be used to construct templates for searches, effectively increasing the sensitivity of the detector to such sources by a large factor. Similarly, the final ringdown of the resulting Kerr black hole is well described by perturbation theory.

However, extracting the most important physics from binary mergers requires us to be able to deal with fully non-linear general relativity as the system spirals together and coalesces. Moreover, a number of people believe that there is a significant event rate for the coalescence of massive black hole systems $\left(\sim 20 M_{\odot}\right) .{ }^{1}$ In this case, LIGO is most sensitive to waves emitted from the strong field regime. Indeed, without some theoretical guidance as to what to expect from this regime, it is possible we may miss these events entirely. ${ }^{2}$

E-mail: saul@astro.cornell.edu 
The goal of developing a general algorithm that can solve Einstein's equations for two black holes has remained elusive, however. All attempts to date have been plagued by instabilities. These instabilities are caused by an interplay of three factors: (1) "constraint-violating modes"; (2) "gauge modes"; and (3) boundary conditions. We will briefly explain what each of these terms means.

Einstein's equations, when written as an initial-value problem for computer solution, can be decomposed into two subsystems of equations: constraint equations that must be obeyed on each spacelike hypersurface, or time slice, and evolution equations that describe how quantities propagate from one hypersurface to the next. An analogous decomposition occurs in electromagnetism, which is naturally split into time-independent (divergence) equations that constrain the fields at a particular time, and time-dependent (curl) equations that determine their evolution. For both electromagnetism and gravitation, the system of equations is overdetermined in the following sense: if the constraint equations are satisfied at some initial time, then the evolution equations guarantee that they will be satisfied at subsequent times. For numerical black hole computations, one typically solves the constraint equations only on the initial time slice, and then uses the evolution equations to advance the solution in time.

However, the set of evolution equations also admits unphysical solutions, the constraint-violating modes. Usually one is not interested in unphysical solutions, but if such a solution grows rapidly with time, any small perturbation (say, caused by numerical errors) that excites this solution will grow and eventually overwhelm the physical solution. Having exact solutions of the equations you are solving that are spurious and swamp the true solution kills any chance of extracting science. Different fromulations of Einstein's equations will have different unphysical solutions. This is one reason why some formulations of Einstein's equations may be better suited for numerical evolution than others.

The coordinate freedom inherent in general relativity means that it is very easy to impose coordinate conditions that lead to numerical instabilities - these are "gauge modes". At the moment, these are not as serious as the constraint-violating modes.

As for the boundary conditions, experience has shown that the kind of conditions we choose and how we implement them can affect the stability of an algorithm enormously.

Instabilities caused by the interplay of these three factors have hampered efforts to solve the related problem of binary neutron stars; only very recently ${ }^{3,4}$ has there been some success in finding stable algorithms. However, black hole evolutions face an additional obstacle that is absent in the case of neutron stars: for neutron stars the gravitational field is everywhere regular, but for black holes one must somehow deal with the physical singularity that lurks inside each hole.

There are two main approaches for handling these singularities. The first is to use the coordinate freedom to choose the coordinates (gauge conditions) to avoid the singularities altogether. Such conditions, however, lead to large gradients in the gravitational field variables near the horizon. These grow exponentially in time and ultimately cannot be resolved by the numerical evolution, causing the code to crash. The alternative approach is to excise the region containing the singularity from the computational domain and evolve only the exterior region. If the excision boundary is placed inside the horizon of the black hole, causality assures us that we do not need to impose a physical boundary condition there.

However, black hole excision is only known to be mathematically well-posed if the evolution equations are hyperbolic with characteristic speeds less than or equal to $c$. In this case, the structure of the equations guarantees that even unphysical modes present in the solution (gauge modes, constraint-violating modes) behave causally and cannot propagate out of the horizon. For many representations of general relativity such as the usual ADM (Arnowitt-Deser-Misner) formulation, the evolution equations are of no mathematical type for which well-posedness has been proven, so the suitability of these formulations for black hole excision must be determined empirically on a case-by-case basis. It is in part for this reason that much attention has been recently focused on hyperbolic representations of Einstein's equations.

It is still unclear whether hyperbolic formulations are computationally advantageous. However, it has been shown that the formulation of the evolution equations can affect stability. For example, some instabilities can be 
eliminated by changing from one formulation of Einstein's equations to another ${ }^{5}$ or by modifying the evolution equations to change the spectrum of unphysical modes. ${ }^{6}$

Note that the decomposition of Einstein's equations into evolution equations and constraints is not unique. For example, one can add any combination of constraints to any of the evolution equations to produce a different decomposition. In the case of electromagnetism, the analogue is to add multiples of $\nabla \cdot \mathbf{E}$ and $\nabla \cdot \mathbf{B}$ to the $\partial \mathbf{E} / \partial t$ and $\partial \mathbf{B} / \partial t$ equations. Using this and similar techniques, there have been a large number of new formulations of $3+1$ general relativity proposed in recent years, ${ }^{5,7-26}$ many of which have attractive properties such as symmetric hyperbolicity.

All such formulations must have the same physical solutions since they describe the same underlying theory. The goal is to overcome the instability problems by finding a suitable reformulation.

\subsection{An Example of a New Formulation of Einstein's Equations}

To illustrate the potential benefits of changing the form of Einstein's equations that one actually integrates numerically, consider the so-called "Einstein-Christoffel" (EC) system. ${ }^{21}$ This is a hyperbolic formulation where the evolution equations look like wave equations in curved spacetime. It is very attractive for doing black hole excision, because no information propagates out of a black hole provided the excision boundary is inside the black hole. A simple test case for the method is to evolve a single Schwarzschild black hole, given the initial data on some time slice. Since the complete solution is known analytically, one has a strong check on the numerical simulation.

When such a situation is evolved in a spherically symmetric coordinate system, the evolution runs forever with arbitrary accuracy. ${ }^{27}$ However, evolving the same initial data with a fully 3 -d code using the EC formulation is unstable. ${ }^{28}$ The code loses all accuracy in a time of about $\sim 30 M$.

As mentioned above, one can change the form of the evolution equations by adding various multiples of the constraints to the right-hand sides. We studied one such generalization of the EC system that has two arbitrary parameters related to what multiples of the constraints are added. We then evolved the same unstable Schwarzschild solution, and studied its stability as we varied the parameters. We found a range of parameters where the instability was much more slowly growing: the evolution ran to $t \sim 600 \mathrm{M}$ before losing accuracy. The result is illustrated in Figure 1. We plot as a function of time the norm of a component of the "momentum" constraint equation, which should be zero. The left panel shows the results for the original EC system for various numerical resolutions. One can see that the code is convergent, with the error approaching machine roundoff at $t=0$ for the highest resolution. But by $t \sim 30 M$ the error has grown so large that the results are not reliable. The right panel shows the same case for the generalized system. Here one sees that the code runs to $t \sim 600 M$ before the error has grown too large. Our best evolutions for this case run to $t \sim 1300 M$.

The significance of these results is that the binary period at the innermost stable circular orbit is expected to be of order $40-100 M$. Thus a code that could evolve a binary for $600 M$ would be adequate to follow the last few orbits and the plunge. Work is proceeding now to see whether these results, obtained for single black hole test cases, still hold for binary evolutions.

\section{NS-NS MERGERS}

The comments in the previous section apply also to NS-NS mergers. However, since excision is not required for neutron stars, solving the field equations is somewhat easier and stable codes exist. Running these codes long enough, with sufficient accuracy, and over a sufficient range of paramters, will tax even the largest supercomputers.

\section{R-MODES IN RAPIDLY ROTATING NEUTRON STARS}

The r-mode instability was discovered by Andersson, ${ }^{29}$ who showed that all rotating, inviscid stars are unstable because of this general relativistic effect. The instability arises when certain stellar oscillation modes, called Rossby waves (or r-modes), are driven unstable by the emission of gravitational waves. As a result, the rotational energy of the star is converted into both mode energy and gravitational waves, causing the star to spin down. 

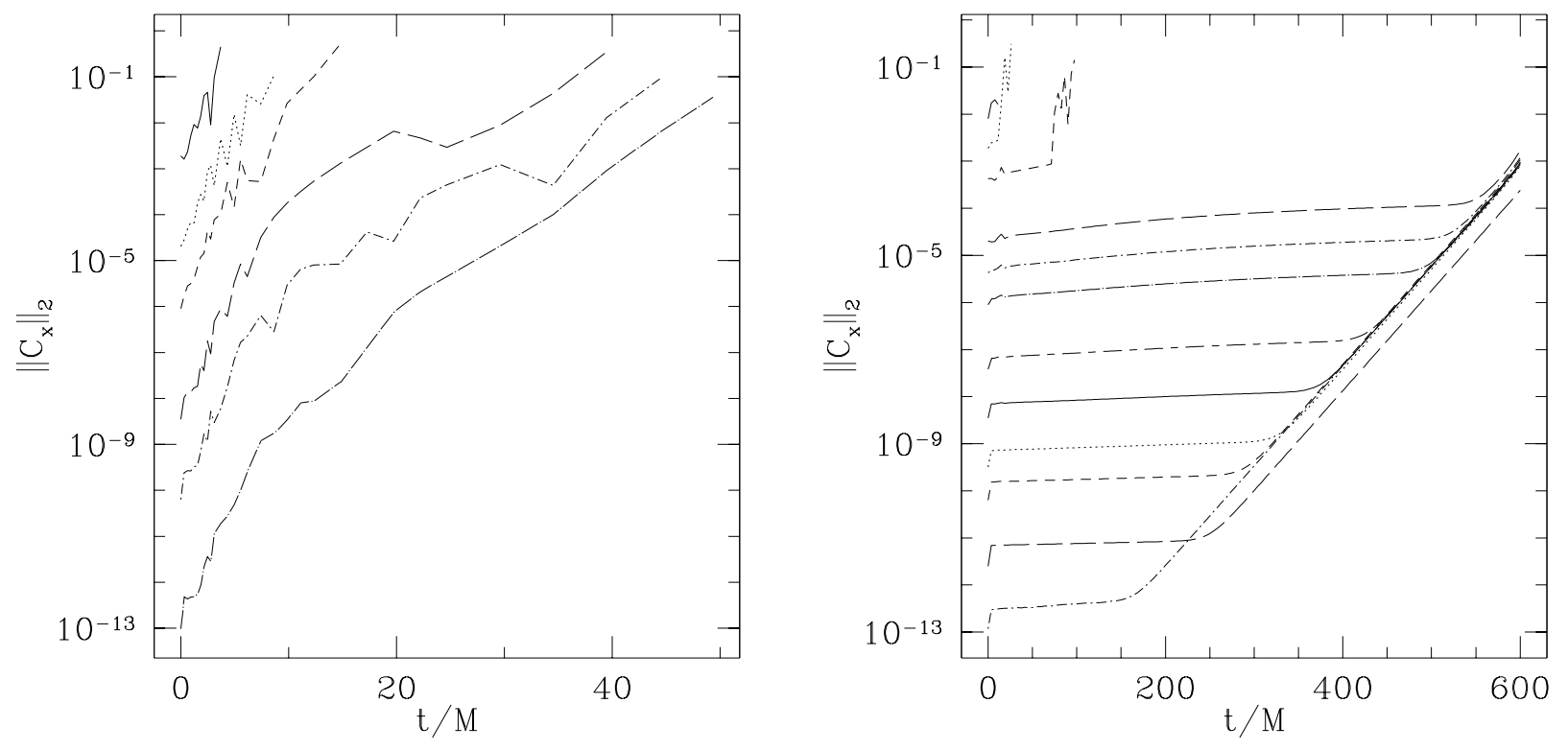

Figure 1. Left: Momentum constraint $\mathcal{C}_{x}$ versus time for evolutions of a Schwarzschild black hole using the EinsteinChristoffel system (see text). Results are plotted for several radial resolutions ranging from 10 to 40 basis functions in a spectral code, fixed angular resolution of 7 spherical harmonics, and fixed time resolution $\Delta t=0.015 M$. Higher radial resolutions correspond to smaller errors. Right: The same evolution shown on the left, but with a careful choice of the arbitrary parameters in the equations. Note that the evolution time scale is about a factor of 20 longer.

Detailed calculations ${ }^{30-33}$ show that viscous dissipation by various mechanisms is insufficient to counter this driving in rapidly rotating neutron stars.

The r-mode instability may be important for two reasons. First, r-modes in any neutron star rotating faster than some critical rate will become unstable, causing the star to rapidly spin down. Hence, r-modes may set a maximum rotation rate for neutron stars. Second, the enormous amount of energy radiated in gravitational waves may be detectable by LIGO.

An open question is how the instability saturates, and at what perturbation amplitude. This question is not only important from a theoretical standpoint, but also must be answered in order to assess whether or not the gravitational radiation emitted during the development of the instability is detectable.

There have been, to date, two fully nonlinear numerical calculations of the development of the instability, but neither completely settles the question of saturation, principally because there are practical limitations on what it is feasible to compute. The first calculation, by Stergioulas and Font ${ }^{34}$ considered the evolution of the fluid inside a neutron star with isentropic equation of state and fixed spacetime geometry. Their calculations began with a large amplitude excitation in the mode expected to be most unstable, the $\ell=m=2 r$-mode, and followed the subsequent hydrodynamics for about 20 stellar rotation periods, during which time no substantial change in the amplitude of the modal excitation was seen, and no evidence for significant nonlinear excitation of other stellar normal modes was detected.

Lindblom, Tohline and Vallisneri ${ }^{35}$ subsequently performed state-of-the-art 3D Newtonian hydrodynamics simulations including a prescription for the radiation reaction force. The only damping mechanism included in the code is numerical viscosity, and of order $128^{3}$ points were used. They were able to follow the linear growth of the r-mode, all the way into the nonlinear regime. Shocks then formed near the surface of the star, rapidly thermalizing the kinetic energy of the mode.

Since the growth of the instability is so slow compared to the dynamical time in the star, they found 


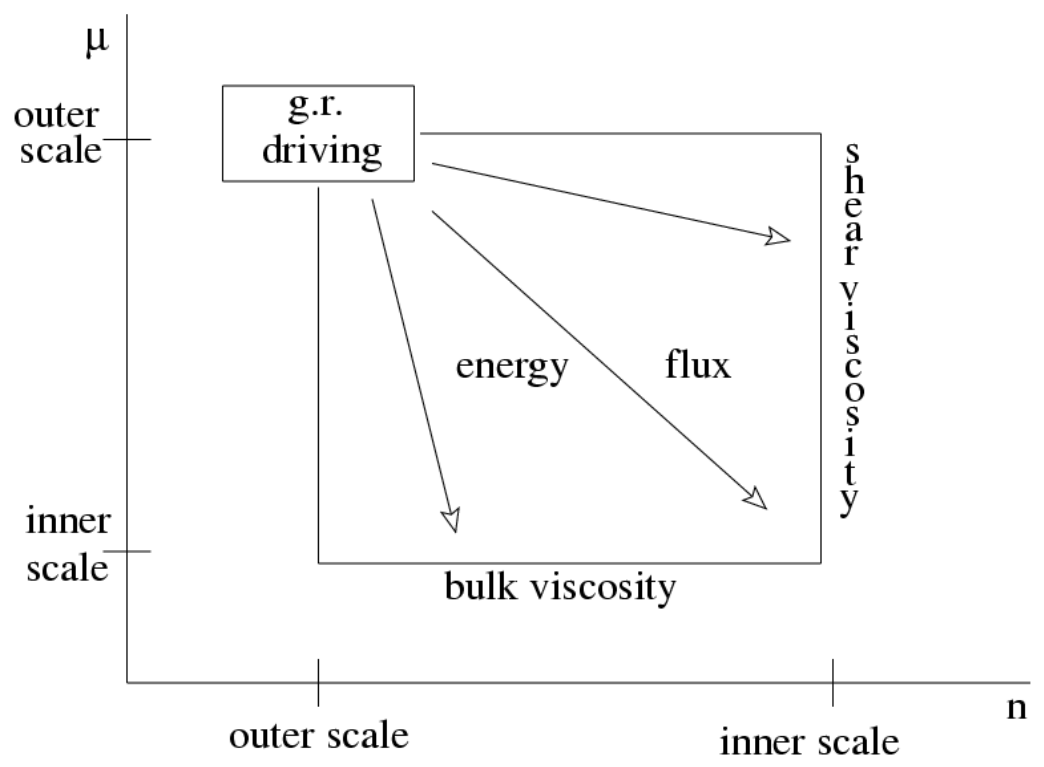

Figure 2. Saturation mechanism for r-modes in rapidly rotating neutron stars. The vertical axis is the rotation frequency of the star. The horizontal frequency is a measure of the mode number of the inertial modes. Energy is input to the r-mode at the outer scale of both $n$ and $\mu$. Energy then cascades to small $\mu$ and large $n$. Bulk viscosity damps away the energy for small $\mu$, and shear viscosity for large $n$.

it necessary to artificially increase the radiation reaction force by a factor of $\sim 4500$. The initial $r$-mode amplitude chosen by Lindblom, Tohline and Vallisneri was small (about 0.1), and, as expected, grew via the radiation-driven instability to large amplitude, where shocks ultimately formed, accompanied by a decrease in $r$-mode amplitude. Both calculations show that nonlinear mode-mode coupling is comparatively weak for $r$-modes, producing little effect even at large modal amplitude on timescales $\sim 10-20$ stellar rotation periods.

A second, more analytical approach to this problem is possible. One assumes, at the outset, that the modes develop only moderately nonlinear amplitudes, and nonlinear effects are modeled perturbatively, via $n$-mode couplings. When amplitudes are small, relatively low order couplings, such as three-mode interactions, suffice, and (when dissipation and radiation reaction are ignored) the equations of motion for the fluid can be modeled as an infinite-dimensional Hamiltonian system with a polynomial interaction potential (which is cubic when only three-mode couplings are kept). This approach has the advantage of allowing explicit calculation of the strength of the lowest order coupling of a given $r$-mode to other modes of the star, but has the disadvantage of failing when mode amplitudes grow very large. Although it is possible that the $r$-mode instability saturates only in the fully nonlinear regime, neither of the numerical calculations proves this with absolute certainty, because it is possible that modal couplings act on timescales longer than covered by the simulations, but shorter than the growth time of the gravitational-radiation instability.

We first developed the tools needed to calculate the nonlinear evolution of the unstable modes in the weakly nonlinear regime. ${ }^{36}$ Next, we worked out a WKB theory of global stellar "inertial" modes. ${ }^{37}$ These are modes like the r-modes in which the Coriolis force is the dominant restoring force. We found that the three-mode interactions can play an important role in damping the r-modes. The reason is that there is a nonlinear transfer of energy to the sea of inertial modes. A turbulent cascade develops, with a constant flux of energy to both large wavenumber, damped by shear viscosity, and small frequency, damped by bulk viscosity. The situation is illustrated in Figure 2.

We can relate these findings to the numerical simulations by considering the following physical example. Imagine water waves being driven by wind moving at $1 \mathrm{~cm} \mathrm{sec}^{-1}$, a whisper of a breeze, as compared to 
$4500 \mathrm{~cm} \mathrm{sec}^{-1}$, a hurricane. For small amplitude water waves, four-wave interactions can transport energy to small scales, saturating the growth of the waves. In a hurricane, the wave growth time is so short that waves grow to large amplitudes and break. Since Lindblom et al. have not addressed how saturation might occur for physical values of either driving or damping of the waves, the relevance of their simulations to saturation of the r-mode instability is not clear.

Our results suggest that their claims of (1) saturation energy of order the rotation energy, and (2) strong shocks as the saturation mechanism, are not supported. They are an artifact of the unphysically large value for the radiation reaction force.

There are some further comments that can be made on the ability of a numerical simulation to accurately

reproduce the cascade of energy to small scales. In simulations with $128^{3}$ points, only a certain number of modes exist because of the finite resolution. It turns out that the energy transfer depends on the existence of nearly resonant modes, that is, a pair of "daughter" modes whose frequencies sum to minus the frequency of the "parent" mode. The number of nodes in each generation of daughter modes is an order of magnitude larger than the parent generation. Current numerical simulations are simply unable to model the energy transfer at all because of their limited resolution.

The consequences of these calculations for neutron star spin evolution are as follows. First, a rapidly rotating $\left(10^{3} \mathrm{~Hz}\right)$ young neutron star can still spin down to a frequency well below the break-up frequency in a timescale of a few thousand years, after which the spindown is dominated by magnetic dipole radiation. Second, we find the spin-up of neutron stars in LMXB's by accretion can still be halted by radiation reaction spindown torque, explaining the observed period clustering in these systems. Lastly, since it would have been difficult to detect gravitational waves from r-modes in young neutron stars even if the saturation amplitude was unity, the small amplitude we have found makes them completely undetectable.

\section{OTHER SOURCES REQUIRING LARGE SCALE COMPUTATIONAL STUDIES}

There are many other important potential sources for LIGO-class detectors that require large-scale numerical simulations to maximize the scientific payoff. A partial list includes

- Tidal disruption of neutron stars by black holes. Advanced LIGO detectors have the possibility to probe the structures of neutron stars and the equation of state of neutron-star matter (at densities up to 10 times nuclear) by observing the tidal disruption of neutron stars by companion black holes.

- Stellar collapse. LIGO has a good possibility of watching two types of stellar collapse. LIGO may see the collapses of stellar cores to form neutron stars, or proto-neutron stars or black holes, including some collapses that trigger supernovae and perhaps others that do not. It may also see the accretion-induced collapse of some white dwarf stars in binaries. The physics of these collapses may be quite complex, including much dirty astrophysics.

- Formation and evolution of $\mathrm{BH} / \mathrm{BH}$ binaries in globular clusters. The most likely source for initial LIGO is the inspiral and merger of stellar-mass $\mathrm{BH} / \mathrm{BH}$ binaries. The range of total masses detectable by initial LIGO interferometers is a few to $1000 M_{\odot}$. Main sequence binaries in the field (in the bulk of a galaxy) are thought to produce $\mathrm{BH} / \mathrm{BH}$ binaries with total masses no larger than roughly 40 or $50 M_{\odot}$. However, heavier binaries may form in globular clusters and other star clusters, by stellar-dynamical processes. Recent simulations of stellar and black-hole interactions in star clusters suggest that the above scenario may play out sufficiently successfully to provide an interesting $\mathrm{BH} / \mathrm{BH}$ rate for LIGO, perhaps even for the initial LIGO interferometers.

\section{ACKNOWLEDGMENTS}

The work described here was carried out with my collaborators, Phil Arras, Eanna Flanagan, Larry Kidder, Sharon Morsink, Mark Scheel, Katrin Schenk, and Ira Wasserman. This work was supported in part by NSF grants PHY-9900672 and PHY-0084729 at Cornell University. 


\section{REFERENCES}

1. S. F. P. Zwart and S. L. W. McMillan, Astrophys. J. 528, L17 (2000).

2. E. E. Flanagan and S. A. Hughes, Phys. Rev. D 58, 4566 (1998).

3. W. Landry and S. A. Teukolsky, gr-qc/9912004.

4. M. Shibata and K. Uryu, Phys. Rev. D 61, 064001 (2000).

5. T. W. Baumgarte and S. L. Shapiro, Phys. Rev. D 59, 024007 (1999).

6. M. A. Scheel et al., Phys. Rev. D 58, 044020 (1998).

7. S. Frittelli and O. Reula, Commun. Math. Phys. 166, 221 (1994).

8. Y. Choquet-Bruhat and J. W. York, Jr., C. R. Acad. Sci., Ser. 1 A321, 1089 (1995).

9. A. Abrahams, A. Anderson, Y. Choquet-Bruhat, and J. W. York, Jr., Phys. Rev. Lett. 75, 3377 (1995).

10. C. Bona, J. Massó, E. Seidel, and J. Stela, Phys. Rev. Lett. 75, 600 (1995).

11. M. H. P. M. van Putten and D. M. Eardley, Phys. Rev. D 53, 3056 (1996).

12. S. Frittelli and O. A. Reula, Phys. Rev. Lett. 76, 4667 (1996).

13. H. Friedrich, Class. Quantum Grav. 13, 1451 (1996).

14. F. B. Estabrook, R. S. Robinson, and H. D. Wahlquist, Class. Quantum Grav. 14, 1237 (1997).

15. M. S. Iriondo, E. O. Leguizamon, and O. A. Reula, Phys. Rev. Lett. 79, 4732 (1997).

16. A. Anderson, Y. Choquet-Bruhat, and J. W. York, Jr., Topol. Meth. Nonlin. Anal. 10, 353 (1997).

17. M. A. G. Bonilla, Class. Quantum Grav. 15, 2001 (1998).

18. G. Yoneda and H. Shinkai, Phys. Rev. Lett. 82, 263 (1999).

19. M. Alcubierre, B. Brügmann, M. Miller, and W.-M. Suen, Phys. Rev. D 60, 064017 (1999).

20. S. Frittelli and O. A. Reula, J. Math. Phys. 40, 5143 (1999).

21. A. Anderson and J. W. York, Jr., Phys. Rev. Lett. 82, 4384 (1999).

22. H. Friedrich and A. Rendall, in Einstein's Field Equations and their Physical Implications, Lecture Notes in Physics, edited by B. G. Schmidt (Springer-Verlag, Berlin, 2000), pp. 127-223.

23. G. Yoneda and H. Shinkai, Int. J. Mod. Phys. D 9, 13 (2000).

24. H. Shinkai and G. Yoneda, Class. Quantum Grav. 17, 4799 (2000).

25. G. Yoneda and H. Shinkai, Class. Quantum Grav. 18, 441 (2001).

26. S. D. Hern, Ph.D. thesis, University of Cambridge, gr-qc/0004036 (1999).

27. L. E. Kidder et al., Phys. Rev. D 62, 084032 (2000).

28. L. E. Kidder, M. A. Scheel and S. A. Teukolsky, Phys. Rev. D 61, 064017 (2001).

29. Andersson, N., Astrophys. J. 502, 708+ (1998).

30. Lindblom, L., Mendell, G., \& Owen, B. J., Phys. Rev. D 60, 064006+ (1999).

31. Bildsten, L. \& Ushomirsky, G., Astrophys. J. Lett. 529, L33 (2000).

32. Levin, Y. \& Ushomirsky, G., Mon. Not. Roy. Astron. Soc. 324, 917 (2001).

33. Lockitch, K. H. \& Friedman, J. L., Astrophys. J. 521, 764 (1999).

34. N. Stergioulas and J. A. Font, Phys. Rev. Lett. 86, 1148 (2001).

35. L. Lindblom, J. E. Tohline, and M. Vallisneri, Phys. Rev. Lett. 86, 1152 (2001).

36. Schenk, A. K., Arras, P., Flanagan, É. É., Teukolsky, S. A., \& Wasserman, I., Phys. Rev. D 65, 024001+ (2002).

37. Arras, P., Flanagan, É. É., Schenk, A. K., Morsink, S. M., Teukolsky, S. A., \& Wasserman, I., astro$\mathrm{ph} / 0202345$ (2002). 